\title{
GSM Based Gas Leak Monitoring System
}

\author{
Asnor Juraiza Ishak ${ }^{1}$, Sarmad Nozad Mahmood ${ }^{2}$, Abadal - Salam T. Hussain ${ }^{3}$ \\ ${ }^{1,2}$ Department of Electrical and Electronic Engineering, Faculty of Engineering, Universiti Putra Malaysia \\ ${ }^{3}$ Department of Medical Device Technologies, Alkitab University Iraq
}

\section{Article Info}

Received Dec 6, 2018

\section{Keyword:}

LPG Gas

Gas Detection System

GSM Monitoring Technique

Gas Leak Control System

\begin{abstract}
This paper used a safe wireless module to detect a gas leakage. The module is household use application where home fires or Liquefied Petroleum Gas (LPG) are classified as the reason of the disasters. The LPG is considered as the most inflammable gases that can ignite fires even in the far distances where gas leak exists. The module can be applied in several places particularly in the fabrications that depend mainly on LPG gas to manage their works. According to the facilities offered by this work, the whole module is functionally separated to perform two tasks identified by gas leak monitoring and the precautions taken accordingly. The module reads the gas sensor in a proposed environment to discover whether gas concentration exceeds a specified range. The system will be activated once the module detects that the gas concentration is altered, and accordingly the control action turns the alarm system alongside with air puller device ON, and sends a warning SMS to a certain recipient using GSM module.
\end{abstract}

\section{Corresponding Author:}

Sarmad Nozad Mahmood

Department of Electrical and Electronic Engineering, Faculty of Engineering

Universiti Putra Malaysia

Serdang, 43400 Seri Kembangan, Selangor, Malaysia

Email: sarmadnmahmood@gmail.com

\section{Introduction}

The LPG can be leaked in different forms such as gaseous or liquid aspects. Generally, a given leak in the liquids are vanished due to the evaporation processes that generates a cloud of gas shown stuck on the wall or ground as it is heavier than the air. The LPG is found widely in the houses, fabrications and fuels. The researchers were struggling in 1910 to investigate the reason of why the gasoline evaporates faster. They discovered that the propane, methane, butane are gases that evaporate so fast. It is worth mentioning that the LPG and the natural gases are environmentally friendly and classified as being detected easily. These kinds of gases are kept in cylinders and can be vanished in ordinary temperature degrees. The LPG gas is considered as a mixture of butane, propane and saturated - unsaturated hydrocarbons $[1,2,3]$. The impact of the LPG on human being is extremely considerable and it can cause burns over the skin, long sleep, annoyed breathing, etc. This risky gas has several individualities such as pressure, flammability, combustion and toxicity. This project is designed to detect and monitor the leak of the gases relatively in the closed places. The module can be employed as household application to supervise and apply control action on different situations accordingly. In addition, the module can be used in the hospitals, hotels, cars and in the industries that use LPG gas as substantial applications. The detection process of the gas in this work excites multiple measurements such as alarm systems, control devices and communication systems based on the Global System for Mobile Communication (GSM) module [4, 5, 6]. 


\section{System Components}

The system is mainly constructed of several components unveiled as follows:
A. Arduino Microcontroller.
B. SIM900 - Arduino GSM Shield Unit.
C. Liquid crystal display unit.
D. MQ-2 Gas sensor module.

\section{A. Arduino Microcontroller}

Arduino microcontroller is an electronic board - open source unit deals with the hardware and software. The Arduino is a friendly board manufactured in different forms such as mini, UNO, Nano, Mega, etc [7, 8]. The whole module is considered as the most compatible microcontrollers with both soft portions as the sensors that work to realize data of a corresponding situation, and either with hard portions such as the motors, actuators and the other sophisticated components. The Atmel microchip technology ATMEGA [9] unit is considered as the main sector seen usually on the top of the board and used to store the programming code to apply the control action on desired environments. To be more specific, the microcontroller unit used in this work and shown in Figure 1 is Arduino UNO.

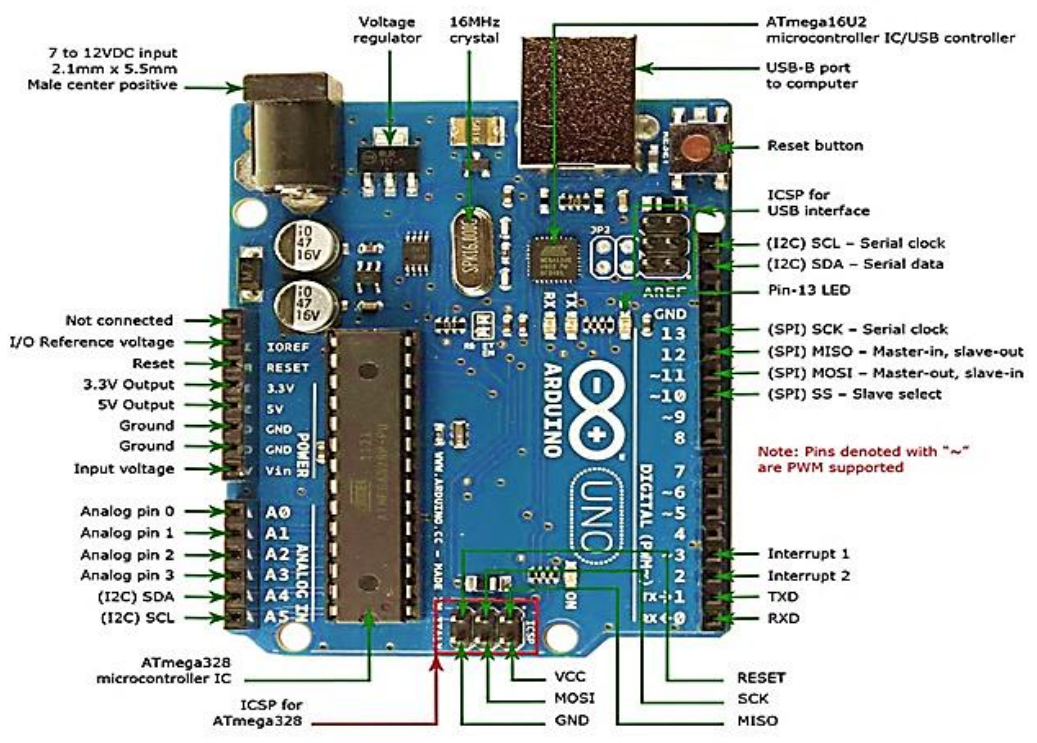

Figure.1. Arduino UNO microcontroller

\section{B. SIM900 - Arduino GSM Shield Unit}

The GSM module is the communication medium used to assign the route of the connection among different locations remotely. The term GSM is the abbreviation given to the global system for mobile communications that is the international station to manage the data transfer processes efficiently. The GSM unit employed in this work is SIM900 Arduino GSM shield that practically known as Quad - Band GSM / GPRS unit. The GSM module shown in Figure 2 offers several services such as Voice, SMS, Data and Fax, knowing that each service is linked with a corresponding frequency branded by $(850,900,1800$, and $1900 \mathrm{MHz})$, respectively [10]. The proposed GSM module works ordinarily with external input voltage around ( $3-5$ volt), classifying the whole unit as the most compatible modems with the friendly microcontrollers such as Programmable Integrated Circuit (PIC), Arduino and Raspberry Pi. The symbol rate of the modem is specified by (9600 115200 bit per second). Hereby, it is intended to assign the range of the communication topology over 900 MHz based on SMS service that makes data broadcasting through mobile phones more applicable. 


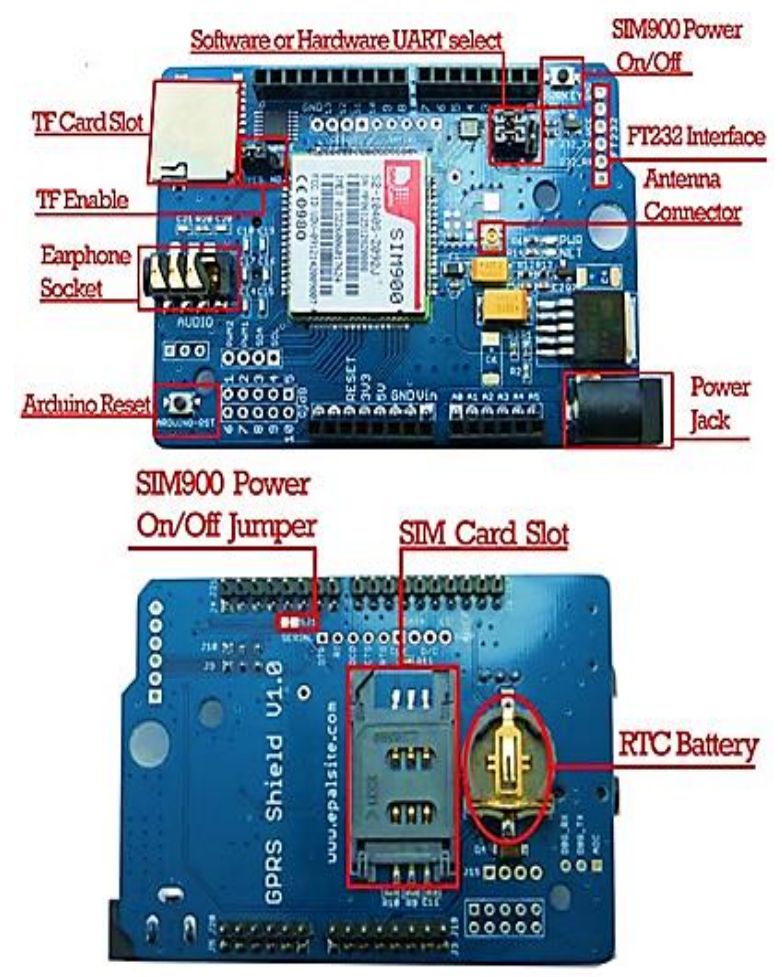

Figure.2. SIM900 GSM Arduino shield

For more specifications, the ports of the module can be clarified as shown in Table 1.

Table 1:. SIM900 GSM ports description

\begin{tabular}{|l|l|}
\hline \multicolumn{1}{|c|}{ PORT NAME } & \multicolumn{1}{c|}{ CLARIFICATION } \\
\hline Power Jack & Connected to 5 - 12 V external power. \\
\hline Micro SD Card & Labeled by SD - EN to enable / disable the card \\
\hline Earphone Socket & $3.55 \mathrm{~mm}$ socket is compatible \\
\hline & SIM900 GSM module can be communicated with \\
& Arduino through the following ports: \\
& $\mathrm{D}_{0}$ : Serial RX of Arduino (Hardware). \\
& $\mathrm{D}_{1}$ : Serial TX of Arduino (Hardware). \\
& $\mathrm{D}_{2}$ : Serial RX of Arduino (Software). \\
& $\mathrm{D}_{3}$ : Serial TX of Arduino (Software). \\
& $\mathrm{D}_{7}$ : Software power, ON/OFF of SIM900. \\
\hline UART Select & Connects any USB with SIM900 through FT232RL \\
\hline FT232RL & SIM900 ON/OFF power button \\
\hline PWRKY & \\
\hline
\end{tabular}

\section{Liquid Crystal Display module}

The liquid crystal display (LCD) unit is a video display that develop light modulation to display several outputs such as picture or text on a screen. The LCD displays have developed considerably to be included in wide variety applications since their inventions in 1964 [11]. In this work, a $16 \times 2$ LCD unit is employed to be assigned as the sight that demonstrates the resultant data. The number $16 \times 2$ is attached to the display module refers to show the data on 16 columns and 2 rows. The proposed LCD module is chosen due to its quite compatibility with the Arduino. The amount of data realized by the sensors are reported through the microcontroller and revealed on the LCD module as information. For simplicity, the connection scheme between the LCD module and the proposed microcontroller which is shown in Figure 3 was done in Fritzing software. 


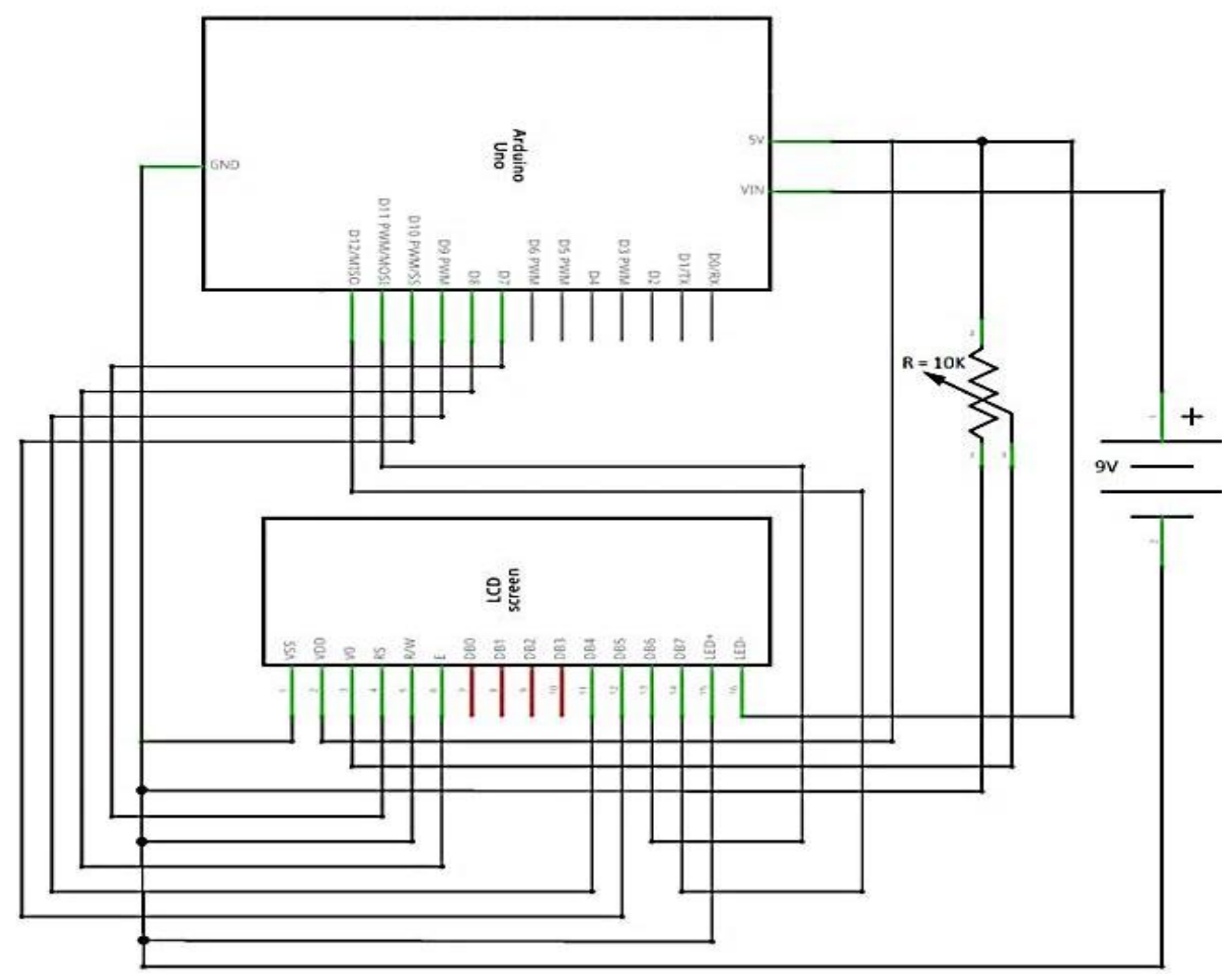

Figure.3. LCD - Arduino connection scheme

The LCD module offers 16 pins [11] were simply clarified as exposed in Table 2.

Table 2: LCD pins task

\begin{tabular}{|c|c|c|}
\hline PIN & TASK & NAME \\
\hline 1 & GND & $\mathrm{V}_{\mathrm{SS}}$ \\
\hline 2 & Provide $5 \mathrm{~V}$ & $\mathrm{~V}_{\mathrm{DD}}$ \\
\hline 3 & Screen light control & $\mathrm{V}_{\mathrm{O}}$ \\
\hline 4 & Register Select & $\mathrm{RS}$ \\
\hline 5 & Read / Write Signal & $\mathrm{R} / \mathrm{W}$ \\
\hline 6 & Signal Enable & $E$ \\
\hline 7 & \multirow{4}{*}{ Low order data bus } & \multirow{4}{*}{$\mathrm{D}_{0}-\mathrm{D}_{3}$} \\
\hline 8 & & \\
\hline 9 & & \\
\hline 10 & & \\
\hline 11 & \multirow{4}{*}{ High order data bus } & \multirow{4}{*}{$\mathrm{D}_{4}-\mathrm{D}_{7}$} \\
\hline 12 & & \\
\hline 13 & & \\
\hline 14 & & \\
\hline 15 & Backlight $5 \mathrm{~V}$ & LED + \\
\hline 16 & Backlight $0 \mathrm{~V}$ & LED - \\
\hline
\end{tabular}

\section{MQ-2 Gas Sensor Module}

Liquefied Petroleum Gas (LPG) or the sensitive material of MQ-2 gas sensor is $\mathrm{SnO} 2$ considered as lower conductivity in the clean air. The conductivity of the sensor shown in Figure 4 gets higher as long as gas concentration is elevated. The sensitivity of MQ-2 sensor is considered exceptionally higher to propane, LPG, hydrogen, methane, and the other steams. Moreover, the cost limitation of the proposed sensor is assigned to 
be appropriate for numerous applications $[12,13]$. The sensor realizes the flammable gas by an increase in the temperature when excited by the heating components. As a working principle, when gas leak is detected the conductivity of the sensor gets higher proportionally with gas concentration raising.

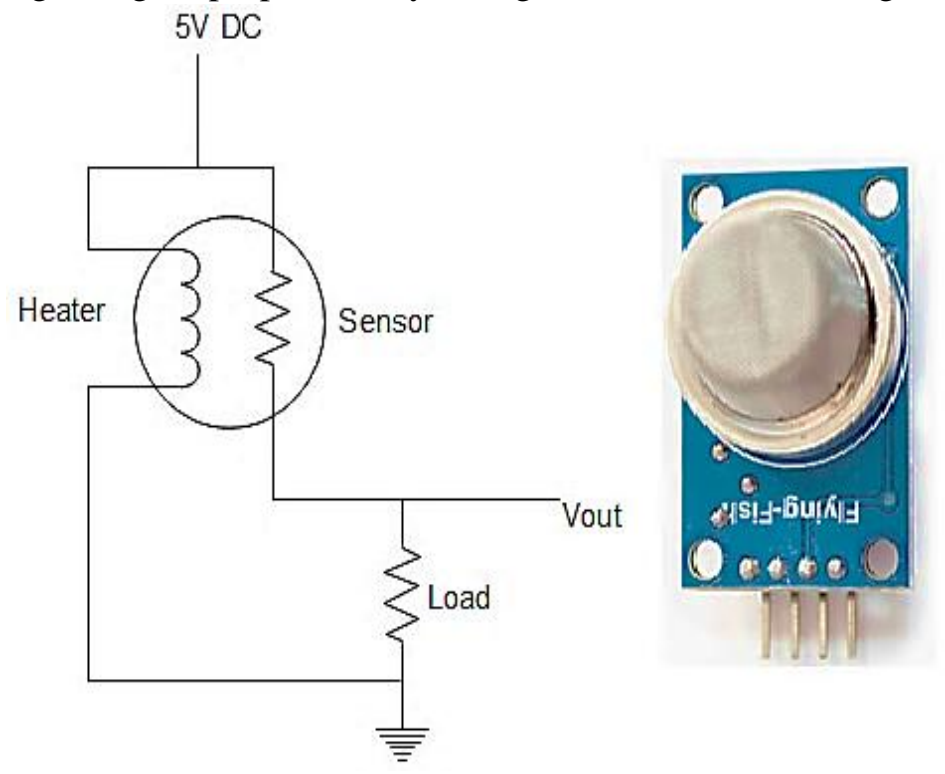

Figure.4. Schematic gas sensor module

In order to improve the performance of the proposed sensor, the load resistance RL should be chosen wisely by fulfilling the following equation:

$\mathrm{P}_{\mathrm{S}}=\frac{V c^{2} * R_{S}}{\left(R_{S}+R_{L}\right)^{2}}$

Where,

$\boldsymbol{P}_{\boldsymbol{S}}$ : Power of sensitivity body.

Vc: Loop voltage.

$\boldsymbol{R}_{\boldsymbol{S}}$ : Sensing resistance.

$\boldsymbol{R}_{L}:$ Load resistance.

As long as, a sensitive resistor is attached in between the output and the GND as shown in Figure 5, the value of the load resistance does not make sense to be restricted. While, the value of the chosen RL is preferred to be around $(2-47 \mathrm{~K} \Omega)$, meaning that the lower value offers less sensitivity, the higher value offers less accuracy.

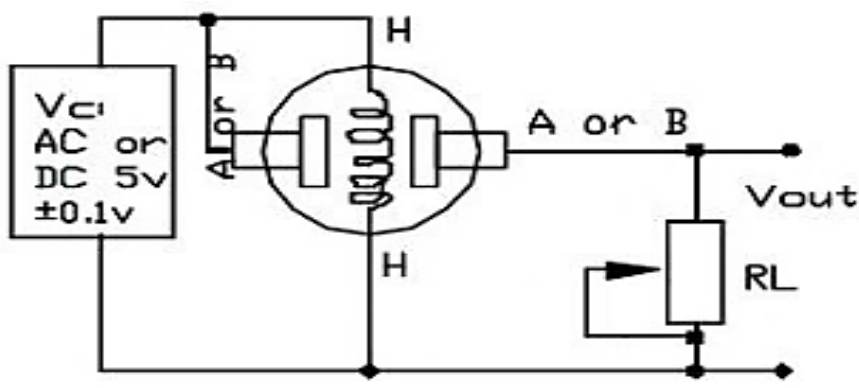

Figure.5. Gas sensor interior construction

Meanwhile, the calibration procedure of the sensor for 1000ppm - LPG or butane concentration in the air can be performed by assigning the value of RL about $20 \mathrm{~K} \Omega[12,14]$. For more simplicity, the connection relation between MQ-2 gas sensor and the Arduino UNO is exposed in Table 3.

Table 3: Arduino vs. MQ - 2 connection table 


\begin{tabular}{|c|c|}
\hline ARDUINO UNO & MQ - 2 GAS SENSOR \\
\hline Any digital pin & $\mathrm{D}_{\text {out }}$ \\
\hline Any analog pin & $\mathrm{A}_{\text {out }}$ \\
\hline $5 \mathrm{~V}$ & $\mathrm{~V}_{\mathrm{CC}}$ \\
\hline GND & GND \\
\hline
\end{tabular}

\section{Gas Leak Control System}

The whole system is proposed to be home-used application under the supervision of the microcontroller with respect to MQ - 2 gas sensor. The control system was designed to serve as monitoring center that detects and applies control action on the realized situation by the sensor module. As a background, the system is considered controllable since it satisfies the block diagram methodology shown in Figure 6.

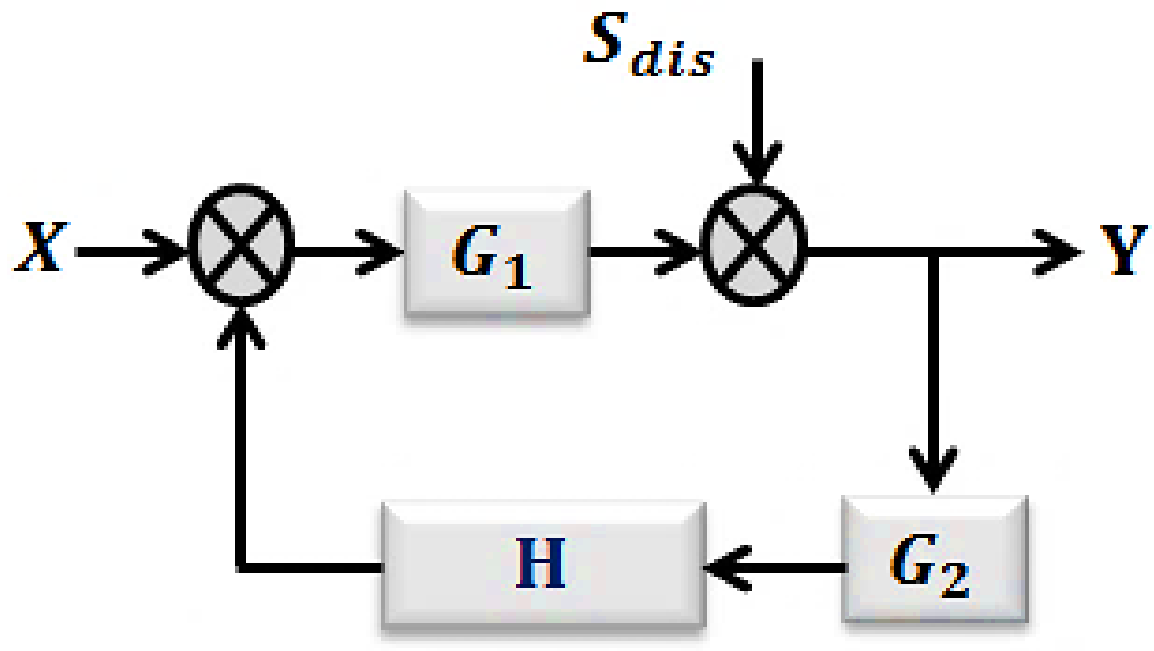

Where,

Figure.6. Control action processes

$\boldsymbol{X}$ : The input signal.

$Y$ : The output signal.

$\boldsymbol{G}_{\mathbf{1}}$ : The uncontrolled system (Plant).

$\boldsymbol{G}_{\mathbf{2}}$ : The processing block (Sensor).

$\boldsymbol{H}$ : The feedback of the system (Controller).

$S_{d i s}$ : The disturbance signal.

The components shown in Figure 6 can be illustrated as the uncontrolled environment $G_{1}$ or the plant as called in control system is the portion that contains all the possible situations that can happen. $S_{d s i}$ is the unexpected signal which can invade the environment that is the term represented by gas leak in this work. $G_{2}$ or the sensor is the part responsible for updating system controller with latest changes in the environment and feed the system back as controller input. Finally, the most popular part is the term H, which leads the whole environment to its quite - desirable condition. In this work the plant would be the house environment that needs to be under the supervision of the controller. The system shown in Figure 7 represents the entire schematic connection and the interaction of the controller with the environmental changes. The proposed module in this work is constructed of Arduino UNO microcontroller, LCD unit, SIM900 GSM shield, Relay module, MQ - 2 gas sensor, and an air puller Fan alongside with a buzzer used as alarm system. The working principle of the module starts normally with no gas leak in the environment, making the control system in an idle situation. Once gas leak is detected, the whole module will be put in charge to start monitoring and supervising the situation. 


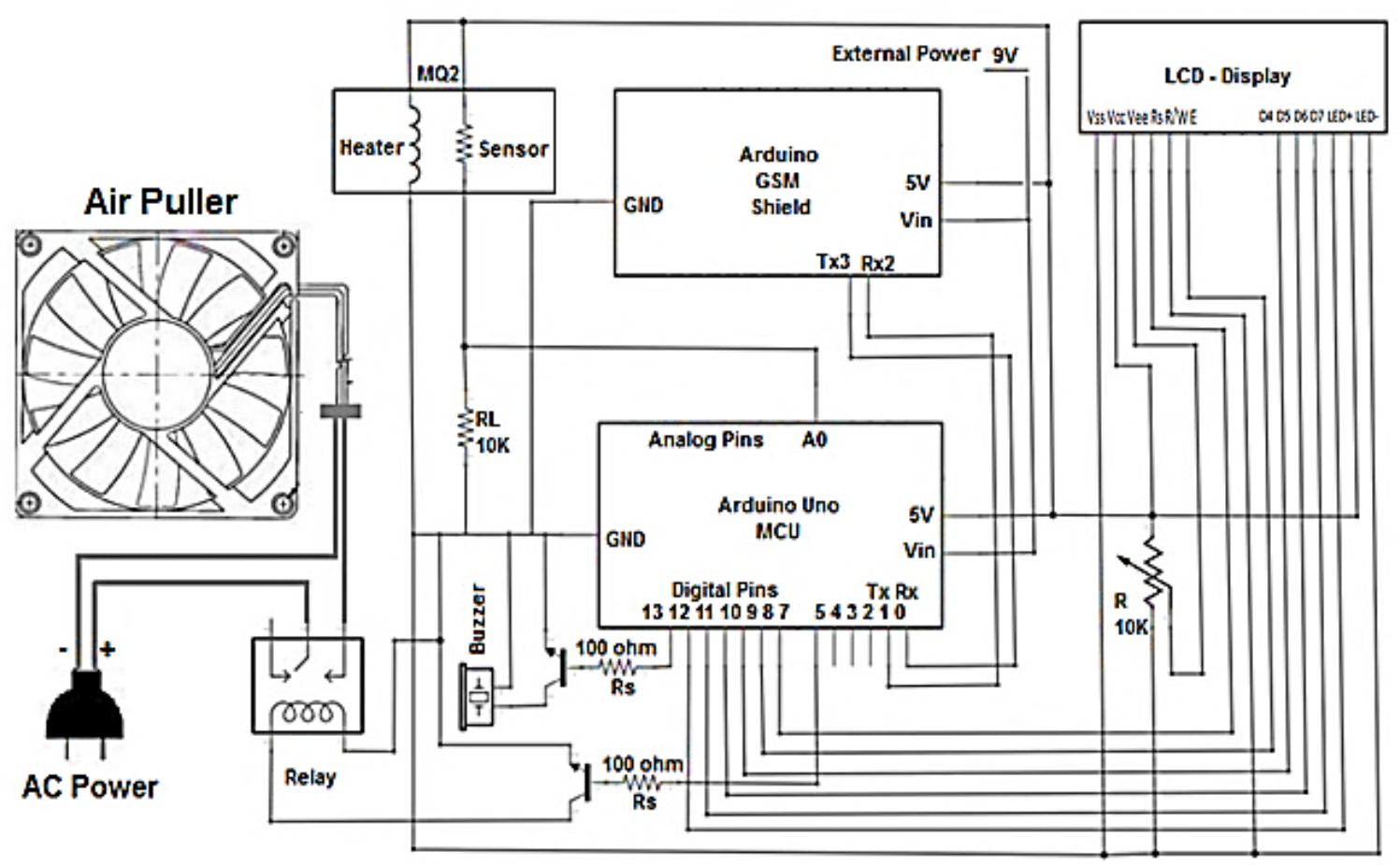

Figure.7. The schematic connection of the module

When the gas sensor is excited by leakage signal the controller triggers the relay module by PWM signal from the microcontroller to take the action by turning the alarm system and the relay ON synchronously in order to allow the AC power to flow through the Fan and pull the gas out of the environment accordingly. Occasionally, the matter was proposed to be vice versa with the elimination of the gas leak problem. Simultaneously, an automatic signal excites the GSM shield to broadcast the condition of the environment as an SMS to a corresponding phone number. Finally, the LCD module is employed in the system in order to demonstrate gas leak situation on the $16 \times 2$ character introducer alongside with the GSM module.

\section{The Code As Flow Chart}

Due to the complexity of the programing code and to excite the direct awareness in the minds, it is intended to show the whole code as a simple flow chart shown in Figure 8. First of all, it has to be reminded that the analog read range for a sensor connected to one of the analog pins in the Arduino is specified by $(0-1023)$. The chart shows that the whole procedure starts by reading the sensor of the gas to decide and discover the situation of the environment. Once reading operation is completed, a decision making procedure starts to apply control action according to a given result. In this work, it is proposed to assign two precautions based on decision results. The first precaution takes place by considering that MQ -2 Sensor stated that gas concentration is greater than 200 as an analog read. In this case the buzzer is turned ON, the air puller works to pull out the amount of the gas spread in the air, the system sends a warning SMS to the specified phone number and simultaneously demonstrates this situation on the LCD module. The second precaution starts with no action applied when it is stated that there is no gas in the air, hence the air puller alongside with the buzzer are turned OFF and the display module states that there is no gas leak detected. 


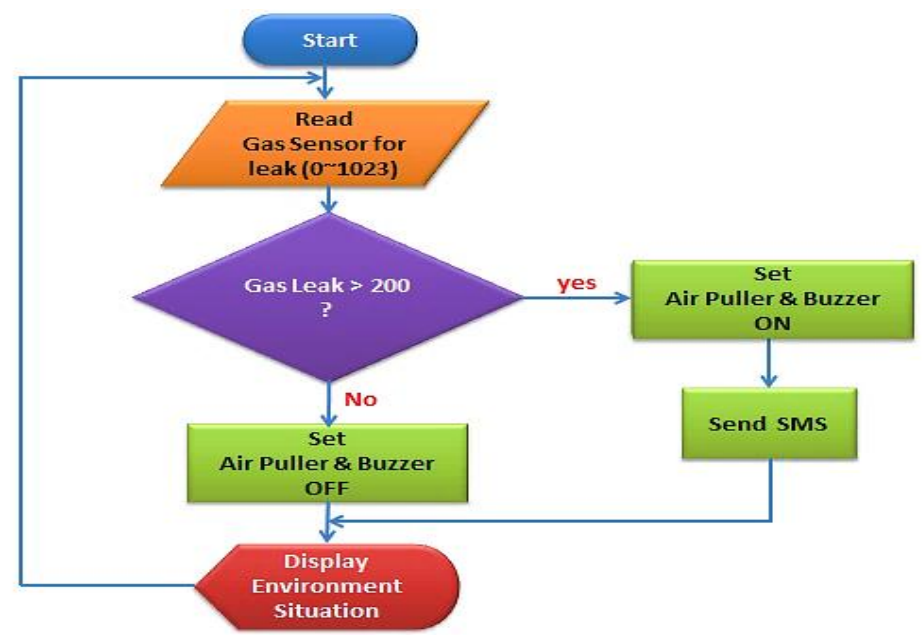

Figure.8. Programming code as flow chart

\section{Conclusions}

This work exhibits a robust friendly organized system design with low cost limitation. The project fascinates the universe for being implemented in the houses, buildings, fabrications and the other closed places. The system applies triple impact authority on the corresponding location due to the dependency on tripartite precautions specified by turning both air puller alongside with the buzzer ON and sending an SMS to a certain phone number. Finally, the control system covers larger areas depending on the GSM module that works under the domination of mobile communication network.

\section{References}

[1] Soundarya, T., et al., C-leakage: Cylinder LPG Gas leakage Detection for home safety. IOSR Journal of Electronics and Communication (IOSR-JECE), 2014. 9(1): p. 53-58.

[2] ÖZMEN, Ramazan, and Mustafa Günay. "An Investigation on Deformation Behaviors of Energy Absorbers for Passenger Coaches." Periodicals of Engineering and Natural Sciences (PEN) 5, no. 3 (2017).

[3] Sharif, Md Haidar, Ivan Despot, and Sahin Uyaver. "A proof of concept for home automation system with implementation of the internet of things standards." Periodicals of Engineering and Natural Sciences (PEN) 6, no. 1 (2018): 95-106.

[4] Fraiwan, L., et al. A wireless home safety gas leakage detection system. in Biomedical Engineering (MECBME), 2011 1st Middle East Conference on. 2011. IEEE.

[5] Mustafa, K. and H. Gitano-Briggs. Liquefied petroleum gas (LPG) as an alternative fuel in spark ignition engine: Performance and emission characteristics. in Energy and Environment, 2009. ICEE 2009. 3rd International Conference on. 2009. IEEE.

[6] Fauadi, Muhammad Hafidz Fazli Md, Suriati Akmal, Mahasan Mat Ali, Nurul Izah Anuar, Samad Ramlan, Ahamad Zaki Mohd Noor, and Nurfadzylah Awang. "Intelligent Vision-based Navigation System for Mobile Robot: A Technological Review." Periodicals of Engineering and Natural Sciences (PEN) 6, no. 2 (2018): 47-57.

[7] Sabharwal, N., et al., A low cost zigbee based automatic wireless weather station with GUI and web hosting facility. 2014, ICRTEDC.

[8] Mahmood, S.N. and F.F. Hasan, Design of Weather Monitoring System Using Arduino Based Database Implementation. Journal of Multidisciplinary Engineering Science and Technology (JMEST), ISSN, 2017: p. 2458-9403.

[9] Andrade, P. B., Cruvinel, P. E., \& Pe, E. A. (2018, January). Module for Virtual Calibration of Sensors of Agricultural Spraying Systems (Temperature, Pressure and flow) Using an Arduino-Based Architecture and a Controller Area Network Bus (CAN). In 2018 IEEE 12th International Conference on Semantic Computing (ICSC) (pp. 352-357). IEEE. 
[10] Nasution, T.H., et al. Electrical appliances control prototype by using GSM module and Arduino. in Industrial Engineering and Applications (ICIEA), 2017 4th International Conference on. 2017. IEEE.

[11] Lapshina, P. D., Kurilova, S. P., \& Belitsky, A. A. (2019, January). Development of an Arduinobased CO2 Monitoring Device. In 2019 IEEE Conference of Russian Young Researchers in Electrical and Electronic Engineering (EIConRus) (pp. 595-597). IEEE.

[12] Nograles, H.A.H., et al. Low cost Internet based wireless sensor network for air pollution monitoring using Zigbee module. in Digital Information and Communication Technology and it's Applications (DICTAP), 2014 Fourth International Conference on. 2014. IEEE.

[13] Machappa, T., M. Sasikala, and M.A. Prasad, Design of Gas Sensor Setup and Study of Gas (LPG) Sensing Behavior of Conducting Polyaniline/Magnesium Chromate (MgCrO \$ $\{4\}$ \$) Composites. IEEE Sensors Journal, 2010. 10(4): p. 807-813.

[14] Geng, L., Li, Y., Shao, S., Liu, D., \& Zhang, Q. (2018, February). The Design of the Intelligent Window Control System Based on Wireless. In 2018 10th International Conference on Measuring Technology and Mechatronics Automation (ICMTMA) (pp. 294-297). IEEE. 